different, more so even than asinous and duct cancer. Dr. Ernest Glynn, in a note on the Detection of anthrax in industrial material, pointed out that the disease appeared to be spreading among domestic animals, and consequently a larger number of agriculturists were becoming infected. It was difficult to demonstrate anthrax spores in industrial material ; two methods were commonly used, namely, agar plate cultures and inoculation of guinea-pigs. The latter method seemed the better, and by its means anthrax spores had been found in 21.3 per cent. of 141 samples of raw hides, wool, etc., examined. Dr. Blair Bell described a new clinical method of estimating the amount of Calcium excreted in the urine It consisted in centrifuging and measuring the amount of calcium oxalate precipitated by oxalic acid, the phos phates being dissolved with acetic acid in a calibrated tube. The estimation was made by comparing the amount precipitated from the urine with that obtained in a similar manner from an artificial standard urine containing known quantity of calcium. Sir JAMES BARR said that the profession was very much indebted to Dr. Blair Bell for the great amount of work which he had unclertaken in order to determine clinical method for accurately estimating the amount of lime in the urine. This apparatus, which he now showed, would fulfil all the necessities of clinical work in respect of estimating the lime salts in the urine. His own method for estimating the amount of lime in the urine was a very simple one. He took an ounce of urine, neutralized it with a little ammonia, then added concentrated solution of oxalic acid with 5 per cent. of strong acetic acid. This precipitated the lime as calcium oxalate, and all the phosphates were dissolved by the acetic acid. 'With some experience the eye soon recog nized what was normal and what was abnormal. He also showed that the excretion cf lime by the lidneys was much greater than was usually supposed. Dr. H. R. Hurter expressed surprise and satisfaction that the results obtained by Dr. "Blair Bell's method had been shown to coincide so closely with quantitative chemical estimations. He suggested the possible employment of a saturated solution of sodium sulphate as an alternative reagent for precipitating the lime salt.

\section{A Correction.}

In the account of the paper on the treatment of diabete mellitus read by Dr. O. T. Williams and Dr..Mildred Powell at the meeting of the Liverpool Medical Institution on March 7 th, and reported in the JoURNAL of March 23rd, it was stated (p. 674, lines 21 to 23 ) that "In the study of the cases treated by (p. 674, lines 21 to 23 ) that "In the study of the cases treated by hydrate metabolism," etc. The report should have stated that "there was no obvious action," etc.

\section{LEEDS AND WEST RIDING MEDICO- CHIRURGICAL SOCIETY.}

At a meeting on March 1st, the President in the chair, Dr. J. B. Hellier, in a paper on Vesicular mole, described two cases in which excessive vomiting in pregnancy had been due to such cause. It was sometimes very difficult to distinguish the malignant form at an early stage from the benign form, the, proliferation of the trophoblastic layer in the two forms respectively being one of degree rather than of kind. Mr. WaLTER Thompson described a case on which he had recently operated successfully for Chorion-epithelioma following vesicular mole. Dr. T. Churton showed a child aged 3, in whom obliteration of the hepatic veins had produced rapidly recurring ascites. This was finally treated by incision, and recovery occurred with dilatation of the superficial abdominal veins. Mr. J. F. Jobson showed a gall-stone removed from the ileum where it had produced acute intestinal obstruction, and also gall bladders which had been in fistu'ous communication with $(a)$ the colon and $(b)$ the duodenum. Mr. Michael A. Teaze showed two siste:s, each of whom had Congenital dislocation of the lens.

MANCHESTER MEDICAL SOCIETY.

At a meeting on March 6th, Dr. E. S. Reynolds, President, in the chair, Dr. W. Fletcher Shaw, in a paper on the Treatment of placenta praevia based upon 100 consecutive cases, said that the best treatmont for both mother and child was to leave Nature alone, provided the haemorrhage was not very great and the doctor could remain in the house until the labour was over. If the haemorrhage were more severe, manual dilatation of the cervix, followed by rapid extraction of the child with forceps or version, gave the best results. Simple podalic version gave excellent results to the mothers, but generally sacrificed the child. Packing, Champetier de Ribes's bag, and Caesarean section he did not advocate for this condition. Dr. C. Paget Lapage, in a paper on certain forms of Fever of obscure origin in infancy and childhood, drew attention to otitis media as one common cause thereof. Fever, when due to blood diseases and Hodgkin's disease, might be either acute or chronic with exacerbations, and in these cases there might be no evidence of the cause until the blood was examined. Infection of the genito-urinary tract with the Bacillus coli communis, either as an acute pyelitis or in a less severe form, was a very important cause, and its existence pointed to the need of careful examination of the urine in infants. Other causes were gastro-intestinal disturbances, such as chronic intestinal indigestion, recurrent fever due to excess of carbohydrates, and even over-feeding with breast milk.

\section{UNITED SERVICES MEDICAL SOCIETY.}

At a meeting on March 13th, Major WAGGeTt, R.A.M.C. (T.), in the chair, Fleet Surgeon A. GASkELL, R.N., in a paper on Dust traps and their dangers on board ships, said that since a ship of war was necessarily overcrowded in point of cubic space per man, the dangers that were represented by dust were even greater on such ships than on shore. There should be a close stucly of hygiene by the officers of the constructive branch of the navy in order that corners and parts difficult to keep clean should ke as far as possible eliminated during the building of the ship. The old rough "cork-paint," which in the past was so freely used as a covering of metallic surfaces, was a great dust collector, but was not in evidence in the most modern ships. The material used at present in covering steel decks and the method followed of laying it were, however, fruitful causes of accumulations of dirt, dust, and damp. He suggested the substitution of asphalte or some other similar material. One point for satisfaction was that ships were now being fitted with disinfectors. They might also be fitted with some form of vacuum cleaner.

\section{HUNTERIAN SOCIETY.}

Ат a clinical meeting at Guy's Hospital on February 28th the interesting proceedings included an $x$-ray demonstration in the medical radiography department by Dr. A. C. Jof.DAN. The cases shown included that of a woman of 32 who, owing to the nature of her pain, was thought to be suffering from gall stones. It was found, however, on $x$-ray examination after a bismuth meal, that the pain was due to a duodenum which was greatly elongated and distended to more than double the normal diameter by kinking at the duodeno-jejunal junction. The duodenum was observed contracting vigorously for seven or eight minutes before it finally succeeded in expelling a portion of its contents into the jejunum through the kink. Subsequent examinations revealed the fact that there was great stasis at the lower end of the ileum, amounting to more than twenty-six hours; this stasis caused the lower coils of the ileum to be dragged down with the caecum into the lowest part of the pelvis. The jejunum was thus pulled upon, with the production of the dcod sno-jejunal kink already described. Thus the duodenal distension was shown to be secondary to the stasis in the ileum.

\section{HARVEIAN SOCIETY.}

AT a meeting on March 14th, Dr. H. J. Macevoy, President, in the chair, Dr. R. H. CoLe defined Hypochondriasis as a subjective state of ill health which was more or less chronic, and was out of all proportion to any disorder of the bodily mechanism. The hypochondriac was one who was painfully conscious of his organic sensations, these 